\title{
Effectiveness of Simulation among Undergraduate Students in the Critical Care Nursing
}

\author{
Melba Sheila D'Souza ${ }^{1}$, Ramesh Venkatesaperumal', Frieda S Chavez ${ }^{2}$, Kader Parahoo ${ }^{3}$ and Deva- \\ kirubai Jacob ${ }^{1}$
}

${ }^{1}$ College of Nursing, Sultan Qaboos University, Oman

${ }^{2}$ Lawrence Bloomberg Faculty of Nursing, Dalla Lana School of Public Health, University of Toronto, Canada

${ }^{3}$ Institute of Nursing and Health Research and School of Nursing, Ulster University, Northern Ireland

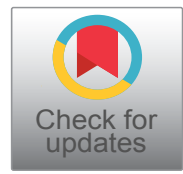

*Corresponding author: Melba Sheila D'Souza, PhD, M.Phil, MSc, RN, Assistant Professor, College of Nursing, Sultan Qaboos University, Muscat, Oman, Tel: 0096898137770,E-mail: melba123@rediffmail.com

\begin{abstract}
Aim: This paper evaluates the effectiveness of simulation in critical care nursing among students in a public university.

Methods: A single quasi-experimental design was used to evaluate the effectiveness of simulation compared to videos and High Fidelity Simulation (HFS). A convenience sample of 100 students in the critical care nursing course was selected in the year 2015-2016. Standardized simulation instruments were used to measure the knowledge, performance, self-satisfaction and self-confidence, educational practices and simulation design among students. ANOVA and t-test was used to analyze the findings.

Results: 'Active learning', 'Diverse ways of learning' and 'High expectations' were significantly higher for satisfaction and confidence among students in learning using simulation compared to videos'. Support', 'Problem solving' and 'Fidelity was significantly higher with Satisfaction and Importance among students using simulation compared to videos.

Conclusion: Knowledge, performance, self-satisfaction and confidence improved among critical nursing students exposed to high fidelity simulation.
\end{abstract}

\section{Keywords}

Nursing, Education, Satisfaction, Self-confidence, Simulation

\section{Highlights}

'Active learning', 'Diverse ways of learning' and 'High expectations' were significantly higher for Self-satisfaction and Self-confidence among students using simulation.

'Support', 'Problem solving' and 'Fidelity' (realism) was significantly higher with Satisfaction and Importance in the simulation among nursing students.
Knowledge, performance, self-satisfaction and self-confidence improved among critical care nursing students exposed to simulation.

\section{Introduction}

Novice nursing students are trained to develop skills for delivering safe patient-centered holistic care in the undergraduate nursing. Entry of novice students in the higher levels of the undergraduate curriculum entails their adult clinical experience in the intensive care, high acuity, post-anesthetic and emergency settings. This clinical practice exposes the students to a variety of clinical conditions with unpredictable socio-cultural and psychosocial background [1]. With growing patient acuity and the complexity of care, higher standards of nursing and competencies are set for new graduates in the clinical settings. Nurse educators endeavor to promote students' critical thinking skills and confidence and are confronted with interactive technology for improving student centered learning $[2,3]$.

Simulations technology constructs real critical care scenarios for improving active participation, clinical reasoning, judgment, and acute decision making skills [4]. It provides hands-on practice and experiences in life-saving measures, resuscitation, cardiac arrest, casualty, disaster and emergency management to enhance understanding and learning. Simulation technology in teaching and science encourages interaction between 
students' feelings and activities and the development of competencies. Example engagement in role play in a simulated scenario in the midst of peers is based on prior knowledge and practices. It can boost students' skills to critically analyze actions and improve decision-making skills $[5,6]$.

Simulated patient care skills can be designed in a structured laboratory for nursing care to improve clinical reasoning [7] and learning outcomes [8]. Use of High Fidelity Simulation (HFS) facilitates transition of critical thinking skills and performance among Arab students into real critical care settings in the Middle Eastern countries. This will advance critical thinking and decision-making skills in a safe and supportive environment [9] and initiate development of core competencies [10]. Registered nurses are role-models for the final year students as they facilitate orientation and teaching-learning in the clinical units [11]. Hence the use of simulation affects student learning outcomes (cognitive, psychomotor, and behavioral) in critical settings is an important aspect of the critical care nursing curriculum in undergraduate unwring program in the Sultanate of Oman.

\section{Background}

Over the past decade, nursing schools have gained momentum in the Middle Eastern countries to meet the changing societal needs, population demands and community needs. Nursing schools have raised the bar of standards of nursing practice by preparing baccalaureate nurses with efficient critical skills for safe practice in the critical settings in the Sultanate of Oman. There has been a need for increasing experiential practice in the critical care units in Oman to update to the clinical expectations and preparing the students to take on the role and responsibilities of Registered Nurses (RNs) in the acute and critical care settings. This application and clinical judgement is necessary for student nurses using high fidelity simulation [12]. Students have high expectations using advanced technology and information technology for learning in Oman $[1,3]$.

Use of HFS will bridge the gap between theory and practice through simulation for escalating active-learning [13]. HFS may increase active engagement through observation, role-play and debriefing as a tool in the educational practices and simulation design [14]. Use of HFS boosts active interaction between students and faculty, exchange of knowledge and performance, cooperation, giving feedback, accentuating time on task, collaborating high expectations, valuing diverse talents and ways of learning [15]. As nurse educators and clinical teachers the curriculum requirements and outcomes emphasize preparation of safe student nurses. There are socio-economic, cultural and gender differences among student nurses and their expectations of the clinical learning environment.

Nursing students have recognized that simulated learning improves problem solving abilities, and psychomotor skills [16], knowledge and situation awareness [17]. Past experiences have demonstrated that students' value simulation in a safe controlled environment compared to direct critical care settings in Oman. Little is known about outcomes of simulation learning among Middle Eastern nursing students' to achieve clinical outcomes in the curriculum. There is a need for collaborative simulation practices for transfer of competencies in a safe effective nursing care in critical care settings in Oman. This study evaluates the effectiveness of HFS compared to videos for improving knowledge, performance, satisfaction and confidence among Omani critical care nursing students. This study will be useful to develop further interventional strategies and simulation designs for nursing students in the critical care nursing curriculum.

\section{Aim}

To evaluate the effectiveness of simulation in among students in the critical care nursing course in the undergraduate nursing program at Oman.

\section{Methods}

\section{Design}

A one-time post-test intervention design was used to examine the effectiveness of simulation using videos and high fidelity simulation in the critical care nursing among Omani students.

Setting and population: One of the schools of nursing in a public university was selected to conduct this study in the accredited Simulation Laboratory (SL) using pre-programmed manikins and advanced technology. The SL has Noelle and Laerdal SimMan 3 Generation human patient simulators. The SL had three main units: simulation, control and observation. The simulation unit consisted of the HFS computerized mannequin with computerized screens and remote mobile camera equipped in a life-like critical nursing unit (e.g. hemodynamic monitoring, lifesaving equipment, resuscitation carts). Microphone, recorder, pre-programmed computerized operator devices in the Control unit. The observation unit has a live-telecast LCD screen displaying the simulation unit. A hundred (100) students registered in the critical care nursing course in the pre-licensure BSc Nursing program in the each academic year 20152016. English is the medium of instruction, assessment, examination and communication in the course, program and college. The course clinical team teaching-learning included specific videos and the High Fidelity Simulation (HFS) for the students in the critical care nursing course.

Sample size and sampling technique: The required sample size for values of $\delta$ in effect size of 0.4 for powers of $80 \%$ at 2 -sided $5 \%$ alpha is 100 to find the mean difference between the two interventions [18]. A convenience sample of 100 nursing students registered in the 
critical care nursing course was used in the study. 100 students who volunteered in this study were exposed to both the videos and HFS in the critical care course in an interval of 8 weeks in the course. These students were tested twice after the videos and the HFS interventions.

Sampling criteria: Inclusion criteria was students confirmed enrollment in the critical care nursing course in the BSc nursing program and provided voluntary participation. Students registered in other clinical courses, with an 'academic withdraw status' or did not volunteer were excluded from the study.

\section{Measurements}

A review of simulation instruments in the public domain illustrated that the National League for Nursing and Laerdal Medical study instruments and simulated scenarios were suitable for the study [19]. Permission was obtained to use the English standardized questionnaires in the study.

Education Practices Questionnaire (EPQ) (student version), a 16-item tool using a 5-point Likert scale processes four educational practices: Active learning, collaboration, high expectations, and diverse ways of knowing in the instructor-developed simulation, and the importance of each practice to the learner. A total score is gained by summing all items, for a total of 80 points each for educational practices and importance subscales. Content validity was established by three nurse educators in simulation. Cronbach's alpha was 0.92 and 0.91 respectively [20]. In our study the internal reliability assessed by Cronbach's alpha was 0.89 and 0.86 for educational practices and importance subscales.

Simulation Design Scale (SDS) is a 20 -item tool with a 5-point Likert scale intended to appraise five design features of instructor-developed simulations. It provides agreement and importance of scenario design: Objectives and information, student support, problem solving, and guided reflection/debriefing/feedback and fidelity. The instrument has two parts: Presence of specific features in the simulation and its importance to the learner. A total score is attained by summing all items, with a possible score of 100 points each on the simulation design and importance subscale. Content validity was established by three nurse educators in simulation. Cronbach's alpha for the overall scale is 0.94 [21]. Internal reliability obtained by Cronbach's alpha was 0.87 and 0.85 for agreement and importance subscales in our study.

Student Satisfaction and Self Confidence in Learning (SSSCL) is a 13-item questionnaire that uses a 5-point Likert scale to measure each item. The instrument measures satisfaction and self-confidence in attaining the instruction for the simulation. Five items are summed for a total score on the satisfaction with current learning scale, with 25 points. A total score is acquired for the self-confidence in learning scale, for a total possible score of 40 points. Cronbach's alpha for satisfaction is 0.94 and self-confidence is 0.87 [22]. Internal reliability measured by Cronbach's alpha was 0.88 and 0.87 for satisfaction and confidence subscales.

Knowledge and performance scenario questionnaire was programmed in the simulated mannequins based on the applied concepts of Myocardial infarction and Cardiac arrest (20 items), Trauma and Shock (20 items) and Diabetes ketoacidosis and Hyperglycemia (20 items) scenarios [23]. It included multiple choice questions with 10 items on knowledge and 10 items on performance with 5 alternative closed responses for each item in each of the three simulation critical care scenarios. There was a total of 60 items with one correct answer for each item got 1 score and the wrong answer gave 0 score. Content validity was established by three nurse educators. Internal reliability got by Cronbach's alpha was 0.81 and 0.82 for knowledge and performance in our study.

Demographic characteristics included age, gender, credits completed, Grade Point Average (GPA) and previous HFS experience.

\section{Quality and integrity of the intervention}

Critical care nursing course has been designed with face-to-face videos and simulation scenarios based on the course requirements, objectives and outcomes developed by the course clinical team. There were no lectures or discussions delivered on these three selected critical care simulated scenarios before the main intervention. 100 students were provided the same interventions including the videos and the HFS. The clinical team monitored the intervention delivery, instrumentation, participant's involvement, ethics and the teaching protocol for both the interventions.

\section{Construction and validation of video intervention}

The clinical team delivered three programmed and standardized scenarios on Myocardial infarction and Cardiac arrest, Trauma and Shock and Diabetes ketoacidosis and Hyperglycemia using videos. The students were exposed to the videos on the selected three scenarios on the first week of the semester in a single classroom setting. Reading materials based on the selected scenarios were offered in the Moodle e-learning system to students before the videos were demonstrated. E-learning pre-reading materials helped the students to participate in the learning. Each video scenario was exhibited for 30 minutes and the total time for the three scenarios was 90 minutes. The pre-test and post-test knowledge and performance scenario questionnaire was done before and after the videos immediately. 100 students who viewed the videos participated in the study.

\section{Construction and validation of simulation intervention}

The Noelle and Laerdal SimMan 3 Generation human patient simulators were pre-programmed to the stu- 
dent's competency levels. The course clinical team developed three dynamic simulations scenarios like Myocardial infarction and Cardiac arrest, Trauma and Shock and Diabetes ketoacidosis and Hyperglycemia to capture the study aim. This included acute and deteriorating patient conditions and evolving symptoms, triggers and cues which responded to the actions of students. The lab technicians managed the simulators and implemented changes in the different parameters in the 15 minutes simulation outline and controlled the patients' voice. These changes were dependent on the students be various in the scenarios. Simulation scenario scripts were roleplayed by standardized performers (actors) as physician, paramedical and family members trained by the course team. Focused reading resources were offered in the e-learning platform based on students' competence and course needs. The validity of the simulated intervention protocol was agreed by two nurse educators experienced in simulation. A trained faculty observed the students' actions. The integrity and fidelity of the intervention was monitored by trained simulated faculty across the two consecutive semesters in the critical care nursing course.

Hundred students experienced the HFS structured simulation scenario in the $8^{\text {th }}$ week of the semester. Students were provided time to be familiar with the simulator and environment and were not aware of the scenarios. They were coached to collaborate as a nursing team, to think aloud and act on the scenario. Student groups were provided detailed study information and instruction kits, intervention and ethical protocols, data collection instruments and informed consent. The students ensured concealment of the simulation scenarios and signed the declaration of confidentiality. Small groups of five student's role played as primary and secondary nurses, physicians, interprofessional collaborative practice in the three simulation scenarios until the selected 50 students completed all the three scenarios.

Pre-briefing was conducted before the real simulated scenario. Each real-time simulation scenario was run for 15 minutes for each of the 10 groups. Simulations were video-taped with informed consent and used for guided reflection. Debriefing was conducted at the end of each scenario with the same group on the same day facilitated by the course team for 20-minutes. It included discussion of outcomes, interventions, priority, decision-making, clinical practice. Observers were random students given specific tasks to focus (e.g. assessment, intervention, collaboration, communication, psychosocial) during the simulated scenario. The simulation lasted for one week. Baseline data consisted of demographic characteristics taken from all the students on day 1 before starting the intervention in the allotted $8^{\text {th }}$ week. Education Practices Questionnaire (EPQ), Simulation Design Scale (SDS) and the Student Satisfaction and Self Confidence in Learning (SSSCL), Pre-test and post-test knowledge and performance scenario questionnaires were administered before and after each simulation scenario.

\section{Ethical considerations and data collection}

University Review Board and College Ethics Committee approval were obtained for the study. Administrative approval was pursued from the Deanship of Student Affairs, Dean and Assistant Deans. Ethical principles and protocols were conformed to the Declaration of Helsinki [24]. Principal investigator and co-investigators maintained credibility, consistency and fidelity of the intervention, data collection, data entry, data cleaning and ethical protocols in the pilot and main study. Informed written and verbal consent was acquired from the students who participated in the study after providing study information and clarifying study details. All the students volunteered to participate in the study in the experimental and the control group. Ethical and research protocols was safeguarded in the study. Participation was voluntary and freedom to withdraw at any time was fortified without affecting student learning. There were no potential risks identified in the study. Students were provided privacy for data collection and feedback as needed. Confidentiality and anonymity was maintained in the study.

\section{Plan for data analysis}

Double data checking was done and password-protected and secured. Data was analyzed using the SPSS 21 software. ANOVA and t-test was used in the study. There were no missing data.

\section{Results}

\section{Demographic characteristics among students}

One-third of the students have completed 101 credits $(35 \%)$ in the BSN program (Table 1 ). Few students (21\%) had been exposed exposure to HFS in the previous courses ( $>5$ times).

\section{Comparison between simulation and videos among nursing students}

Mean overall satisfaction $(77.6 \pm 4.9)$ and self-confidence $(87.6 \pm 4.0)$ and respective subscales were

Table 1: Demographic characteristics among Omani nursing students $\mathrm{N}=100$.

\begin{tabular}{|l|l|l|}
\hline Student characteristics $\mathbf{N}=\mathbf{1 0 0}$ & Variables & Percentage \\
\hline Age & $<20$ years & 48 \\
\hline Gender & $>$ 20 years & 52 \\
\hline & Male & 26 \\
\hline Credits completed & Female & 74 \\
\hline & 50 & 30 \\
\hline & $51-100$ & 35 \\
\hline Grade Point Average (GPA) & $101-130$ & 35 \\
\hline & $<2.50$ & 31 \\
\hline & $2.51-3.00$ & 40 \\
\hline High Fidelity Simulation (HFS) & $>3.01$ & 29 \\
\hline experience & $<4$ times & 79 \\
\hline & $>5$ times & 21 \\
\hline
\end{tabular}


Table 2: Comparison between learning via simulation and videos among students in critical care nursing $N=100$.

\begin{tabular}{|c|c|c|c|c|}
\hline & HFS $N=100$ & Videos $N=100$ & t value & $p$ value \\
\hline \multicolumn{5}{|l|}{ Educational practices } \\
\hline Overall satisfaction & $77.6 \pm 4.9$ & $56.9+6.3$ & 0.44 & 0.25 \\
\hline Active learning & $4.1 \pm 0.3$ & $3.0+0.4$ & 2.1 & $0.03^{*}$ \\
\hline Collaboration & $4.2 \pm 0.6$ & $3.4+0.5$ & 1.43 & 0.31 \\
\hline Diverse ways of learning & $4.3 \pm 0.4$ & $3.2+0.5$ & 1.64 & $0.04^{*}$ \\
\hline High expectations & $4.2 \pm 0.5$ & $3.3+0.5$ & 0.51 & $0.03^{*}$ \\
\hline Overall self-confidence & $87.6 \pm 4.0$ & $66.9+5.3$ & 0.34 & 0.5 \\
\hline Active learning & $4.4 \pm 0.5$ & $3.0+0.8$ & 2.1 & $0.04^{*}$ \\
\hline Collaboration & $4.5 \pm 0.6$ & $3.4+1.4$ & 1.43 & 0.41 \\
\hline Diverse ways of learning & $4.3 \pm 0.7$ & $3.2+2.5$ & 1.64 & $0.05^{*}$ \\
\hline High expectations & $4.1 \pm 0.5$ & $3.3+1.5$ & 0.51 & $0.04^{*}$ \\
\hline \multicolumn{5}{|c|}{ Stud satisfaction and self-confidence } \\
\hline Satisfaction in current learning & $4.2 \pm 0.3$ & $3.5+0.3$ & 3.040 & $0.03^{*}$ \\
\hline Confidence & $4.7 \pm 0.3$ & $3.0+0.3$ & 2.12 & 0.09 \\
\hline \multicolumn{5}{|l|}{ Simulation design } \\
\hline Overall Satisfaction agreement & $88.6 \pm 4.2$ & $64.5 \pm 0.2$ & 2.20 & 0.19 \\
\hline Objectives and information & $4.1 \pm 0.4$ & $3.8 \pm 0.6$ & 3.23 & 0.88 \\
\hline Support & $4.0 \pm 0.4$ & $3.8 \pm 0.3$ & 1.44 & $0.05^{*}$ \\
\hline Problem solving & $4.1 \pm 0.6$ & $3.8 \pm 0.7$ & 1.86 & $0.05^{*}$ \\
\hline Feedback/guided reflection & $4.0 \pm 0.6$ & $3.0 \pm 0.7$ & 1.09 & 0.28 \\
\hline Fidelity (realism) & $4.3 \pm 0.7$ & $3.1 \pm 0.6$ & 0.98 & $0.02^{*}$ \\
\hline Overall Importance & $78.6 \pm 4.2$ & $54.5 \pm 0.2$ & 2.20 & 0.29 \\
\hline Objectives and information & $4.5 \pm 0.4$ & $3.4 \pm 0.6$ & 3.23 & 0.08 \\
\hline Support & $4.0 \pm 0.6$ & $3.8 \pm 0.6$ & 3.44 & $0.04^{*}$ \\
\hline Problem solving & $4.4 \pm 0.6$ & $3.8 \pm 0.7$ & 2.86 & $0.05^{*}$ \\
\hline Feedback/guided reflection & $4.0 \pm 0.5$ & $3.3 \pm 0.7$ & 1.09 & 0.39 \\
\hline Fidelity (realism) & $4.4 \pm 0.7$ & $3.1 \pm 0.8$ & 1.98 & $0.02^{*}$ \\
\hline
\end{tabular}
$p<0.05$.

Table 3: Differences in post-test knowledge and performance scores among students using simulation and videos $\mathrm{N}=100$.

\begin{tabular}{|c|c|c|c|c|}
\hline & High fidelity simulation $\mathrm{N}=100$ & Videos $\mathrm{N}=100$ & $\mathbf{F}$ & p value \\
\hline \multicolumn{5}{|c|}{ Scenario 1: Myocardial infarction and cardiac arrest $N=100$} \\
\hline Pre-test score $(0-20)$ & $7.4 \pm 2.2$ & $6.0 \pm 2.4$ & 0.52 & $0.04^{*}$ \\
\hline Post-test score (0-20) & $15.9 \pm 1.7$ & $10.1 \pm 1.7$ & & \\
\hline \multicolumn{5}{|c|}{ Scenario 2: Shock and trauma $\mathrm{N}=100$} \\
\hline Pre-test score $(0-20)$ & $7.9 \pm 2.2$ & $8.5 \pm 2.4$ & 2.98 & $0.05^{*}$ \\
\hline Post-test score $(0-20)$ & $16.0 \pm 1.2$ & $11.4 \pm 1.4$ & & \\
\hline \multicolumn{5}{|c|}{ Scenario 3: Diabetes ketoacidosis and hyperglycaemia $\mathbf{N}=100$} \\
\hline Pre-test score (0-20) & $8.3 \pm 1.7$ & $7.6 \pm 1.3$ & 2.1 & $0.02^{*}$ \\
\hline Post-test score (0-20) & $17.1 \pm 1.3$ & $12.9 \pm 1.4$ & & \\
\hline
\end{tabular}
* $p<0.05$.

higher among students exposed to the HFS compared to the videos (Table 2). Active learning, diverse ways of learning and high expectations were significantly higher with self-satisfaction and self-confidence in the use of HFS among students compared to videos.

Satisfaction in current learning $(P<0.03)$ was significantly higher among students in the HFS compared to the videos.

Mean overall satisfaction $(88.6 \pm 4.2)$ and importance (78.6 \pm 4.2$)$ was higher among students in the HFS group. Support, Problem solving and Fidelity (realism) was significantly higher with satisfaction and importance in the HFS compared to the videos among nursing students.

Pre and post-test knowledge and performance scenarios
Mean post-test scenario scores was higher in the students exposed to HFS for Myocardial infarction and cardiac arrest (15.9 \pm 1.7$)$, Shock and trauma (16 \pm 1.2$)$ and DKA and hyperglycaemia (17.1 \pm 1.3$)$ compared to the videos (Table 3). Mean post-test scores of the three simulation scenarios were significantly higher with the use of HFS compared to the videos among the students.

\section{Discussion}

Our study confirmed 'Overall Self-satisfaction' and 'Self-confidence' in the 'Educational practices' and higher subscale scores among students exposed to the HFS compared to the videos independent of the roles during simulation and observation. The self-confidence scores were proportionally higher than the satisfaction scores. Educational practices subscales were 'Active learning', 'Diverse ways of learning' and 'High expectations', which 
were significantly higher for Self-satisfaction and Selfconfidence in the use of HFS. Interactive simulation in a safe environment [25] and yield differences in simulation compared to traditional learning [26]. Simulation improves clinical judgement $[27,28]$ while nurse educators facilitate engagement in discussions for active learning in Oman [3]. This indicated positive satisfaction with the simulation lab learning environment and learning through observation, experiences and practice. It direct to the diverse learning styles, experiences and expectations among students in using simulated pedagogy. This shows that students were able to apply knowledge, practical skills and display competencies.

'Satisfaction' and 'Confidence' was demonstrated higher especially with Satisfaction in current learning was among students using HFS compared to the videos in our study. Improvements in simulation were seen compared to lectures and MCQ in critical care [29] and higher satisfaction scores [30]. Simulation improved self-confidence [31,32], while Omani students prefer behavioural competencies and hands-on practices [33].

'Overall Satisfaction and Importance' in the Simulation design was displayed higher among students in the HFS group among students as role players and observers in our study. They study illustrates 'Support', 'Problem solving' and 'Fidelity' (realism) was significantly higher in this study with Satisfaction and Importance in the HFS compared to the videos among nursing students. Feedback from simulation improved reflective learning and critical thinking [34] and performance in the premises of learning among Omani students [14,35]. Thus students displayed guided reflection and discussion of actions as they learn through peers.

In our study Post-test knowledge and performance scores explained significantly higher in the students exposed to HFS in the three scenarios. The post-test scores increased across the scenarios from Myocardial infarction and cardiac arrest, Shock and trauma to the DKA and hyperglycaemia compared to the videos. Higher performance among intensive care nurses in simulated emergency scenarios [36] and increased critical thinking, clinical judgement, skill acquisition, clinical reasoning [37-39]. Simulation translated application in knowledge $[40,41]$ with use of clinical scenarios [42]. Increased post-test simulated scores on assessment and learning complex patient scenarios [43] and increased behaviors scores [44] improved in simulated teaching. Hence knowledge scores improved with higher satisfaction with current learning and self-confidence $[45,46]$. This shows that knowledge is related to performance in the scenarios for applied learning and is closely linked to each other.

Higher age, GPA, credits and prior exposure to HFS among students had higher knowledge and performance. Cohort performance had improvements with simulated practices [47] and higher GPA increased competencies
[48]. Higher age, GPA and number of clinical courses were significant with satisfaction with clinical learning environments in Oman [35].

\section{Limitations}

There was only one group of students and no control group to assess the effectiveness of students' transfer of skills into clinical practice. The study could not compare the demographic differences between the students in the two interventions.

\section{Conclusion}

This study demonstrates improved knowledge and performance among critical care nursing students with use of high fidelity simulation compared to videos. Students perceived higher satisfaction and self-confidence in the safe controlled environment. This is the first study in Oman to confirm that students' learn in complex simulated environments and integrated into nursing course. 'Active learning', 'Diverse ways of learning' and 'High expectations' subscales had significantly higher mean scores among students. It illustrated 'Support', 'Problem solving' and 'Fidelity' (realism) was significantly higher in this study with Satisfaction and Importance in the HFS compared to the videos among critical care nursing students. Students valued satisfaction with current learning and confidence with exposure to the HFS. Use of HFS provides incomparable practice in safe environment to improve knowledge and performance in nurturing critical care nursing concepts and nursing care. Use of HFS technology was valued by critical care nursing students as the simulation was proven effective and beneficial to learning, acquisition of critical skills in a non-risk and safe lab environment. Use of HFS improved self-satisfaction and self-confidence in the as an effective tool for educational practices and simulation design. Students' role play adds to the satisfaction and confidence in various learning situations to develop competencies and improve the abilities to contribute to knowledge and performance.

\section{References}

1. D'Souza MS, Karkada SN, Castro R (2014) Exploring e-learning among nurse educators in undergraduate nursing. Journal of Nursing Education and Practice 4: 73-84.

2. Wotton K, Davis J, Button D, Kelton M (2010) Third-year undergraduate nursing students' perceptions of high-fidelity simulation. J Nurs Educ 49: 632-639.

3. D'Souza MS, Isac C, Venkatesaperumal R, Nairy KS, Amirtharaj A (2014) Exploring nursing student engagement in the learning environment for improved learning outcomes. Clinical Nursing Studies 2: 1-16.

4. Alexander M, Durham CF, Hooper JI, Jeffries PR, Goldman N, et al. (2015) NCSBN Simulation Guidelines for Prelicensure Nursing Programs. Journal of Nursing Regulation 6: 39-42.

5. Norman G, Dore K, Grierson L (2012) The minimal relationship between simulation fidelity and transfer of learning. Med Educ 46: 636-647. 
6. Garrett BM, MacPhee M, Jackson C (2011) Implementing high-fidelity simulation in Canada: Reflections on 3 years of practice. Nurse Educ Today 31: 671-676.

7. Lapkin S, Levett-Jones $T$, Bellchambers $H$, Fernandez $R$ (2010) Effectiveness of patient simulation manikins in teaching clinical reasoning skills to undergraduate nursing students: A systematic review. Clinical Simulation in Nursing 6: e207-e222.

8. Elfrink VL, Kirkpatrick B, Nininger J, Schubert C (2010) Using learning outcomes to inform teaching practices in human patient simulation. Nurs Educ Perspect 31: 97-100.

9. Valler-Jones T, Meechan R, Jones H (2011) Simulated practice-a panacea for health education? $\mathrm{Br} J$ Nurs 20 : 628-631.

10. Ricketts B (2011) The role of simulation for learning within pre-registration nursing education - $A$ literature review. Nurse Educ Today 31: 650-654.

11. D'Souza MS, Jose J, Al-Jabri SS (2013) Perceptions of teaching undergraduate students among registered nurse preceptors. European Journal of Scientific Research 111: 289-299.

12. Lestander O, Lehto N, Engstrom A (2016) Nursing students' perceptions of learning after high fidelity simulation: Effects of a Three-step Post-simulation Reflection Model. Nurse Educ Today 40: 219-224.

13. Swenty CF, Eggleston BM (2011) The evaluation of simulation in a baccalaureate nursing program. Clinical Simulation in Nursing 7: e181-e187.

14. D‘Souza MS, Arjunan P, Venkatesaperumal R (2017) High fidelity simulation in nursing education. International Journal of Health Sciences and Research 7: 340-353.

15. Reese CE, Jeffries PR, Engum SA (2010) Learning together: Using Simulations to develop nursing and medical student collaboration. Nurs Educ Perspect 31: 33-37.

16. Harder BN (2010) Use of simulation in teaching and learning in health sciences: A systematic review. J Nurs Educ 49: 23-28.

17. Cooper S, Kinsman L, Buykx P, McConnell-Henry T, Endacott R, et al. (2010) Managing the deteriorating patient in a simulated environment: Nursing students' knowledge, skill and situation awareness. J Clin Nurs 19: 2309-2318.

18. Chan YH (2003) Randomised Controlled Trials (RCTs)-Sample size: The magic number? Singapore Med J 44: 172-174.

19. Jeffries PR, Rizzolo MA (2006) Designing and Implementing Models for the Innovative Use of Simulation to Teach Nursing Care of III Adults and Children: A National, MultiSite, Multi-Method Study. Summary Report.

20. Hayden JK, Smiley RA, Alexander M, Kardong-Edgren S, Jeffries PR (2014) Supplement: The NCSBN National Simulation Study: A longitudinal, randomized, controlled study replacing clinical hours with simulation in prelicensure nursing education. Journal of Nursing Regulation 5: 1-64.

21. Groom JA, Henderson D, Sittner BJ (2014) NLN/Jeffries simulation framework state of the science project: Simulation design characteristics. Clinical Simulation in Nursing 10: 337-344.

22. Franklin AE, Burns P, Lee CS (2014) Psychometric testing on the NLN Student Satisfaction and Self-Confidence in Learning, Simulation Design Scale, and Educational Practices Questionnaire using a sample of pre-licensure novice nurses. Nurse Educ Today 34: 1298-1304.
23. Kardong-Edgren S, Adamson KA, Fitzgerald C (2010) A review of currently published evaluation instruments for human patient simulation. Clinical Simulation in Nursing 6: e25-e35.

24. Anon (2013) World Medical association declaration of Helsinki. JAMA 310: 2194.

25. Bradley C (2011) The role of high-fidelity clinical simulation in teaching and learning in the health professions. King's College London 33-42.

26. Rode JL, Callihan ML, Barnes BL (2016) Assessing the value of large-group simulation in the classroom. Clinical Simulation in Nursing 12: 251-259.

27. Hallin K, Backstrom B, Haggstrom M, Kristiansen L (2016) High-fidelity simulation: Assessment of student nurses' team achievements of clinical judgment. Nurse Education in Practice 19: 12-18.

28. Lavoie P, Pepin J, Cossette S (2015) Development of a post-simulation debriefing intervention to prepare nurses and nursing students to care for deteriorating patients. Nurse Educ Pract 15: 181-191.

29. Solymos O, O'Kelly P, Walshe CM (2015) Pilot study comparing simulation-based and didactic lecture-based critical care teaching for final-year medical students. BMC Anesthesiol 15: 153.

30. Wang AL, Fitzpatrick JJ, Petrini MA (2013) Comparison of two simulation methods on chinese BSN students' learning. Clinical Simulation in Nursing 9: e207-e212.

31. Ahn H, Kim HY (2015) Implementation and outcome evaluation of high-fidelity simulation scenarios to integrate cognitive and psychomotor skills for Korean nursing students. Nurse Educ Today 35: 706-711.

32. Kaddoura M, Vandyke O, Smallwood C, Gonzalez KM (2016) Perceived benefits and challenges of repeated exposure to high fidelity simulation experiences of first degree accelerated bachelor nursing students. Nurse Education Today 36: 298-303.

33. D'Souza MS, Venkatesaperumal R, Radhakrishnan J, Balachandran S (2013) Engagement in clinical learning environment among nursing students: Role of nurse educators. Open Journal of Nursing 3: 25-32.

34. Murray BA (2014) The use of high-fidelity simulation in psychiatric and mental health nursing clinical education. International Journal of Health Sciences Education 2: 1-12.

35. D'Souza MS, Karkada SN, Parahoo K, Venkatesaperumal R (2015) Perception of and satisfaction with the clinical learning environment among nursing students. Nurse Educ Today 35: 833-840.

36. Randi Ballangrud, Mona Persenius, Birgitta Hedelin, Marie Louise Hall-Lord (2014) Exploring intensive care nurses' team performance in a simulation-based emergency situation, -expert raters' assessments versus self-assessments: An explorative study. BMC Nurs 13: 47.

37. Bussard ME (2015) High-Fidelity Simulation to teach accountability to prelicensure nursing students. Clinical Simulation in Nursing 11: 425-430.

38. Fisher D, King $L$ (2013) An integrative literature review on preparing nursing students through simulation to recognize and respond to the deteriorating patient. J Adv Nurs 69: 2375-2388.

39. Powell-Laney SK (2011) The use of human patient simulators to enhance the clinical decision making of nursing students. Dissertation Abstracts International Section A: Humanities and Social Sciences 71: 3544. 
40. Strickland HP, March AL (2015) Longitudinal impact of a targeted simulation experience on high-stakes examination outcomes. Clinical Simulation in Nursing 11: 341-347.

41. Sperling J, Clark S, Kang Y (2013) Teaching medical students a clinical approach to altered mental status: Simulation enhances traditional curriculum. Med Educ Online 18: 1-8.

42. Chapelain P, Morineau T, Gautier C (2015) Effects of communication on the performance of nursing students during the simulation of an emergency situation. J Adv Nurs 71: 2650-2660.

43. Beckford DMHM (2013) Utilizing simulation curriculum to decentralize mental health concepts. Open Journal of Nursing 3: 227-229.

44. Deborah D Garbee, John T Paige, Laura S Bonanno, Vadym V Rusnak, Kendra M Barrier, et al. (2013) Effectiveness of teamwork and communication education using an interprofessional high- fidelity human patient simulation critical care code. Journal of Nursing Education and Practice 3: 1-12.

45. Thidemann IJ, Soderhamn O (2013) High-fidelity simulation among bachelor students in simulation groups and use of different roles. Nurse Educ Today 33: 1599-1604.

46. Nickless LJ (2011) The use of simulation to address the acute care skills deficit in pre-registration nursing students: A clinical skill perspective. Nurse Educ Pract 11: 199-205.

47. Krautscheid LC (2008) Improving communication among healthcare providers: Preparing student nurses for practice. Int J Nurs Educ Scholarsh 5: 40.

48. Shin H, Sok S, Hyun KS, Kim MJ (2015) Competency and an active learning program in undergraduate nursing education. J Adv Nurs 71: 591-598. 